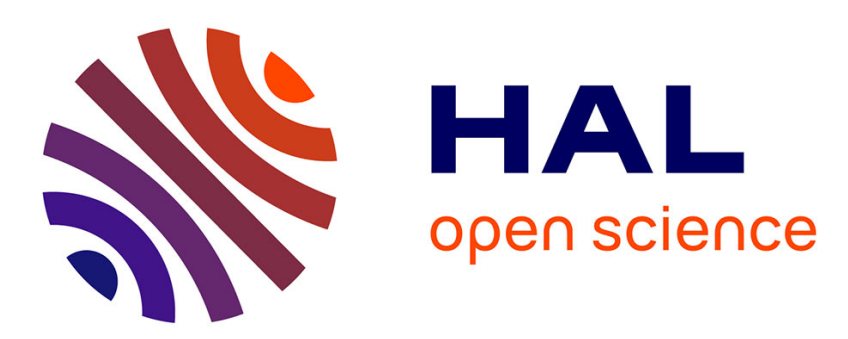

\title{
Exact solution of the totally asymmetric simple exclusion process: Shock profiles
}

\author{
Bernard Derrida, S. A. Janowsky, J. L. Lebowitz, E. R. Speer
}

\section{To cite this version:}

Bernard Derrida, S. A. Janowsky, J. L. Lebowitz, E. R. Speer. Exact solution of the totally asymmetric simple exclusion process: Shock profiles. Journal of Statistical Physics, 1993, 73 (5-6), pp.813-842. 10.1007/BF01052811 . hal-03282965

\section{HAL Id: hal-03282965 https://hal.science/hal-03282965}

Submitted on 19 Jul 2021

HAL is a multi-disciplinary open access archive for the deposit and dissemination of scientific research documents, whether they are published or not. The documents may come from teaching and research institutions in France or abroad, or from public or private research centers.
L'archive ouverte pluridisciplinaire HAL, est destinée au dépôt et à la diffusion de documents scientifiques de niveau recherche, publiés ou non, émanant des établissements d'enseignement et de recherche français ou étrangers, des laboratoires publics ou privés. 


\title{
Exact Solution of the Totally Asymmetric Simple Exclusion Process: Shock Profiles
}

\author{
B. Derrida, ${ }^{1}$ S. A. Janowsky, ${ }^{2,3}$ J. L. Lebowitz, ${ }^{4}$ and E. R. Speer ${ }^{2}$
}

\author{
Received March 3, 1993
}

\begin{abstract}
The microscopic structure of macroscopic shocks in the one-dimensional, totally asymmetric simple exclusion process is obtained exactly from the complete solution of the stationary state of a model system containing two types of particles-"first" and "second" class. This nonequilibrium steady state factorizes about any second-class particle, which implies factorization in the one-component system about the (random) shock position. It also exhibits several other interesting features, including long-range correlations in the limit of zero density of the second-class particles. The solution also shows that a finite number of second-class particles in a uniform background of first-class particles form a weakly bound state.
\end{abstract}

KEY WORDS: Asymmetric simple exclusion process; shock profiles; secondclass particles; Burgers equation.

\section{INTRODUCTION}

Much attention has focused recently on nonequilibrium model systems consisting of particles on a lattice, evolving microscopically under conservative dynamical rules, and described on the macroscopic level by a continuous density field which satisfies some equations of hydrodynamic type $^{(1,2)}$ Of particular interest are cases in which the macroscopic equa-

\footnotetext{
'Service de Physique Théorique, CEN Saclay, F-91191 Gif-sur-Yvette, France. E-mail: derrida@amoco.saclay.cea.fr

${ }^{2}$ Department of Mathematics, Rutgers University, New Brunswick, New Jersey 08903. E-mail: speer@math.rutgers.edu

${ }^{3}$ Present address: Department of Mathematics, University of Texas, Austin, Texas. E-mail: janowsky@math.utexas.edu

${ }^{4}$ Departments of Mathematics and Physics, Rutgers University, New Brunswick, New Jersey. E-mail: lebowitz@math.rutgers.edu
} 
tions produce shocks. Such models usually have microscopic transition rates which do not satisfy any detailed balance condition; that is, the dynamics is not reversible with respect to any measure. In this paper we provide an analytic description of the shock structure of one such model, the much studied one-dimensional, totally asymmetric simple exclusion process, whose macroscopic behavior is described by the Burgers equation, by obtaining an exact solution for a related model system. Before explaining these models in detail we describe briefly their context within nonequilibrium statistical mechanics.

For systems with dynamics not satisfying detailed balance, there is no general formula of the Gibbs type for the steady states. As a result, we do not in general have even qualitative information about the nature of these states or their dependence on the parameters entering the dynamics. Almost all of our information about such driven diffusive systems comes from computer simulations or from some approximate calculations using renormalization group ideas or field-theoretic methods. ${ }^{(3)}$ These indicate the existence of generic long-range correlations in dimensions greater than one, ${ }^{(3-5)}$ even when the dynamics is short ranged. This situation is in strong contrast to the behavior when the dynamics satisfies detailed balance; in this case the steady states are essentially Gibbs states for some shortranged Hamiltonian and therefore have exponential decay of correlations in the weak-coupling or high-temperature regime.

One of the simplest models of driven diffusive systems is the asymmetric simple exclusion process, ${ }^{(6)}$ in which particles on the one-dimensional lattice $\mathbb{Z}$ attempt at random times to jump to an adjacent site, choosing the site to their right with some fixed probability $p$ and that to their left with probability $1-p$, with the attempt succeeding if the target site is not already occupied. Here the Bernoulli measure $v_{\rho}$, in which each lattice site is occupied independently with probability $\rho(0 \leqslant \rho \leqslant 1)$, is a (translationinvariant) stationary state for any value of $p$; in this state there is a current $J=(2 p-1) \rho(1-\rho)$. The dynamics satisfies detailed balance with respect to $v_{\rho}$ if and only if $p=1 / 2$. (In fact, the model may be formulated in any dimension and always has product measures as invariant states; in this regard it is not typical of driven diffusive systems.)

For the asymmetric simple exclusion process the mass density $u(x, t)$ on the macroscopic level-which is an appropriately scaled continuum limit of the particle density in the microscopic model-is described ${ }^{(7-9)}$ by the inviscid Burgers equation

$$
u_{t}+(2 p-1)(u[1-u])_{x}=0
$$

It is well known that solutions of this equation can exhibit shocks (for $p \neq 1 / 2)$. In the simplest such situation the macroscopic density $u(x, t)$ 
satisfies $u(x, t)=u_{+}$for $x>x_{0}(t)$ and $u(x, t)=u_{-}$for $x<x_{0}(t)$, where $u_{+}>u_{-}$(when $p>1 / 2$ ) and the shock position $x_{0}(t)$ moves with the constant velocity $(2 p-1)\left(1-u_{+}-u_{-}\right)$.

An important question to ask of any model which produces shocks is whether the sharpness of the density transition persists on the microscopic scale. In the asymmetric simple exclusion process this problem has been extensively studied both theoretically and numerically. ${ }^{(10-17)}$ In either case one must first locate the shock - that is, define precisely its position-on the microscopic level. This may be done by introducing into the system a special second-class particle, ${ }^{(12)}$ which evolves by a modified dynamical rule chosen to keep the second-class particle near the shock; the position of this particle may then conveniently be taken as the definition of the (microscopic) shock location.

With this definition of shock location it has been established ${ }^{(15)}$ (see also refs. 11,13 , and 14 for related results) that the shock remains sharp on the microscopic level--i.e., that the (ensemble-averaged) particle density as viewed from the second-class particle has a time-invariant distribution which approaches the densities $\rho_{ \pm}=u_{ \pm}$at $\pm \infty$. Computer studies of this shock profile in the case $p=1$ are described in ref. 16 (see also ref. 17). These indicate that in general the density approaches its asymptotic value exponentially fast. In the limiting case $\rho_{+}=\rho_{-}=\rho$, however, when the invariant measure on the original particles (now called first-class particles) is Bernoulli and the density in a fixed frame is thus constant and equal to $\rho$, the choice to view the system from the second-class particle produces a "shock" profile for which the approach to $\rho$ is governed by a power law.

In this paper (see also ref. 18) we study these questions for the totally asymmetric model (TASEP) in which all jumps are to the right, that is, in which $p=1$; for this system we derive exact formulas for the shock density profiles discussed above. (In the conclusion we comment on extensions to more general models.) Our method is to study a model containing arbitrary numbers of first- and second-class particles, ${ }^{(14)}$ which we call the two-species TASEP. In particular, we first obtain an explicit expression for the invariant measure of the two-species TASEP on a ring of $N$ sites. This solution, which is based on an earlier exact solution ${ }^{(19)}$ (see also ref. 20) derived for a finite system with open boundaries and only first-class particles, expresses the probability of any configuration as the trace of a product of matrices or operators.

From this solution on the ring we then study shock profiles and other phenomena by taking the infinite-volume limit $N \rightarrow \infty$ in various ensembles. We consider three main cases. The shock profile between distinct densities $\rho_{+}$and $\rho_{-}$is studied-via an artifice ${ }^{(14)}$ - through the infinite-volume limit of systems with fixed, nonzero densities of both first- and second-class 
particles. On the other hand, the "shock" profile for the constant-density case is obtained from the infinite-volume limit of systems containing one second-class particle and a fixed density $\rho$ of first-class particles. Finally, we study also a pair of second-class particles in the presence of a finite density of first-class particles, and find that they form a bound state.

In the next section we describe more explicitly the two-species TASEP and the method we use to obtain shock profiles on the infinite line from the exact solution of the model on the ring. We derive there a simple symmetry property of the shock profiles and discuss as well several general properties of the invariant measures. We also describe the organization of the remainder of the paper, where the exact solution is obtained and its consequences in the infinite-volume limit are explored.

\section{DESCRIPTION OF THE MODEL AND SUMMARY OF RESULTS}

We begin by describing the two-species TASEP on a ring of $N$ lattice sites, with $K_{1}$ first-class particles, $K_{2}$ second-class particles, and $K_{0}=$ $N-K_{1}-K_{2}$ empty sites. No two particles are allowed to occupy the same site, so that we may specify a configuration of the system by an $N$-tuple $\left(\tau_{1}, \tau_{2}, \ldots, \tau_{N}\right)$, where $\tau_{i}=1$ if there is a first-class particle at site $i, \tau_{i}=2$ if there is a second-class particle at site $i$, and $\tau_{i}=0$ if site $i$ is empty. We treat the site index as cyclic, so that $\tau_{N+i}=\tau_{i}$.

The system evolves under a stochastic dynamical rule. During a time interval $d t$ each adjacent pair of sites in the system-say sites $i$ and $i+1-$ has probability $d t$ of being selected for a possible exchange of their current states. The exchange takes place if (a) site $i$ is occupied by a first-class particle and site $i+1$ is either occupied by a second-class particle or is empty, or (b) site $i$ is occupied by a second-class particle and site $i+1$ is empty; that is, the possible transitions are

$$
10 \rightarrow 01, \quad 12 \rightarrow 21, \quad \text { and } \quad 20 \rightarrow 02
$$

Note that the number of particles of each type is conserved. It is easy to see that any configuration can evolve into any other, so that the system is ergodic and there is a unique stationary probability measure.

In Section 3 we derive an explicit expression for the probability of any configuration in this invariant measure; the remainder of the paper explores the implications of this representation for the behavior of the system in the infinite-volume limit. Although it is possible to carry out the analysis using the canonical ensembles introduced above, it simplifies the computations to introduce superpositions of these-that is, grand 
canonical ensembles-in which the populations of particles can fluctuate, adjusting the fugacities of the first- and second-class particles and of the holes to produce some fixed densities of the various species.

In Section 4 we consider a system which contains a single second-class particle and a fixed density $\rho$ of first-class particles: $K_{2}=1,\left\langle K_{1}\right\rangle / N=\rho$; the quantity which has a nontrivial infinite-volume limit is the density of first-class particles as seen from the second-class particle. In this relative frame the location of the second-class particle is taken to be $i=0$ and we let $d_{i}$ denote the average density of first-class particles at sites $i \neq 0$. The average drift velocity of the second-class particle in a fixed frame is $1-2 \rho$, as we show at the end of this section (see also ref. 15). Because this drift velocity decreases with $\rho$, the effect of density fluctuations is that the second-class particle is attracted toward regions in which the local density has a positive gradient. Thus the density profile has $d_{i}>\rho$ for $i>0$ and $d_{i}<\rho$ for $i<0$. We find that $d_{i}$ converges monotonically to $\rho$ as $|i|$ increases and, as indicated in the introduction, $\left|d_{i}-\rho\right| \simeq C|i|^{-1 / 2}$ for $|i| \gg 1$.

We could, of course, take a infinite-volume limit with any constant number of second-class particles. In Section 5 we consider the case $K_{2}=2$, $\left\langle K_{1}\right\rangle / N=\rho$. Somewhat surprisingly, the two second-class particles form a bound state, with a power-law decay $r^{-3 / 2}$ of the probability of finding them a distance $r$ apart; the distance between them thus has infinite expectation.

A time-invariant state in the ring geometry cannot support two regions of distinct densities of first-class particles. It is possible, however, to compute the density profile in the infinite system for the shock between asymptotic densities $\rho_{+}$and $\rho_{-}$(with $\rho_{+}>\rho_{-}$) by taking the infinitevolume limit from the ring geometry at fixed densities $\rho_{1}=\rho_{-}$of first-class particles and $\rho_{2}=\rho_{+}-\rho_{-}$of second-class particles. Again we suppose that we look at the system relative to one second-class particle, located at the origin; let us denote by $\delta_{i}$ the average density of first-class particles at site $i$, for $i<0$, and the average density of all particles- both first and second class -at site $i$, for $i>0$. Because the second-class particles ahead of the distinguished one behave toward it as if they were first-class particles, it is easy to see that $\delta_{i}$ is exactly the profile we seek. (This is a special case of a method of ref. 14 for identifying a shock profile in a system of first-class particles from the knowledge of a stationary measure for a system with uniform densities of first- and second-class particles, viewed from a second-class particle. The general method works for any asymmetry in the ASEP - that is, for any value of $p$, not just $p=1$.) The calculations for this situation are carried out in Section 6, and the shock profile is discussed in Section 7. We find that $\delta_{i}$ converges exponentially to $\rho_{ \pm}$as $|i| \rightarrow \pm \infty$ and 
that the characteristic length of this decay diverges as $\left(\rho_{+}-\rho_{-}\right)^{-2}$ when $\left(\rho_{+}-\rho_{-}\right) \searrow 0$.

There are several simple general properties of the invariant states of the two-species model. One of these, derived immediately below, is a symmetry property-symmetry around a second-class particle, in the two-component system, or symmetry of the shock, in the one-component system:

$$
\delta_{-i}+\delta_{i}=\rho_{-}+\rho_{+}
$$

Another, derived at the end of Section 3 as a simple consequence of our representation of the invariant measure, is that in the infinite system a second-class particle has the effect of decoupling the regions on each side of it; thus if we condition (as in the discussions above) on the presence of such a particle at the origin and let $f(\tau)$ and $g(\tau)$ be functions which depend only on the occupation variables $\tau_{i}$ for $i<0$ and $i>0$, respectively, then

$$
\left\langle f g \mid \tau_{0}=2\right\rangle=\left\langle f \mid \tau_{0}=2\right\rangle\left\langle g \mid \tau_{0}=2\right\rangle
$$

(A similar independence holds for finite systems in the grand canonical ensemble for expectations conditioned on the presence of at least two second-class particles at specified sites.) A third property depends for its derivation on the somewhat elaborate computations from the explicit formula for the invariant measure which will be given later (see Sections 6 and 7): if we again condition on the presence of a second-class particle at the origin in the infinite system with densities $\rho_{1}$ and $\rho_{2}>0$ of first- and second-class particles, then the distributions of first-class particles to the right of the origin and of holes to the left of the origin are Bernoulli, with densities $\rho_{1}$ and $1-\rho_{1}-\rho_{2}$, respectively.

The derivation of the symmetry (2.2) is based on the fact ${ }^{(6)}$ that the invariant measure for the TASEP at density $\rho$ with only first-class particles is Bernoulli. Let us introduce new occupation variables $\xi_{i}$ and $\zeta_{i}$ taking values 0 and 1 , where $\xi_{i}=1$ (respectively $\zeta_{i}=1$ ) if and only if there is a first-class (respectively second-class) particle at site $i$. Now the second-class particles appear to the first-class particles to be indistinguishable from holes; thus the first-class particles considered separately have a Bernoulli distribution at density $\rho_{1}$. On the other hand, we may ignore the distinction between the two types of particle as well as interchanges $\left(\begin{array}{lll}1 & 2 \rightarrow 21\end{array}\right)$ between them; these new particles themselves form a TASEP system which will have an invariant measure which is Bernoulli with density $\rho_{1}+\rho_{2}$. Thus for all $j \neq k$,

$$
\left\langle\xi_{j} \xi_{k}\right\rangle=\rho_{1}^{2} \quad \text { and } \quad\left\langle\left(\xi_{j}+\zeta_{j}\right)\left(\xi_{k}+\zeta_{k}\right)\right\rangle=\left(\rho_{1}+\rho_{2}\right)^{2}
$$


If we write

$$
\left\langle\left(\xi_{j}+\zeta_{j}\right)\left(\xi_{k}+\zeta_{k}\right)\right\rangle=\left\langle\xi_{j} \xi_{k}\right\rangle+\left\langle\xi_{j} \zeta_{k}\right\rangle+\left\langle\zeta_{j}\left(\xi_{k}+\zeta_{k}\right)\right\rangle
$$

then (2.4) implies that

$$
\rho_{2}^{-1}\left\langle\xi_{j} \zeta_{k}\right\rangle+\rho_{2}^{-1}\left\langle\zeta_{j}\left(\xi_{k}+\zeta_{k}\right)\right\rangle=2 \rho_{1}+\rho_{2}
$$

Since $\rho_{2}^{-1}\left\langle\xi_{j} \zeta_{k}\right\rangle$ is the probability of a first-class particle at site $j$, conditioned on there being a second-class particle at site $k$, and $\rho_{2}^{-1}\left\langle\zeta_{j}\left(\xi_{k}+\zeta_{k}\right)\right\rangle$ is the probability of a first- or second-class particle at site $k$, conditioned on there being a second-class particle at site $j$, for $j<k$ this is just (2.2) with $i=k-j$.

The fact that we can view the two-species TASEP as a one-species TASEP in two different ways also yields a simple argument that in a uniform one-component TASEP at density $\rho$ the velocity of a single second-class particle is $1-2 \rho$. For, if a small density $d \rho$ of second-class particles is added to such a system, then the new system may be regarded as a one-component TASEP at density $\rho+d \rho$ and hence the current must increase from $\rho(1-\rho)$ to $(\rho+d \rho)(1-\rho-d \rho)$. On the other hand, since the first-class particles alone form a TASEP, they still contribute a current $\rho(1-\rho)$, and the additional current due to the second-class particles is $(1-2 \rho) d \rho$.

\section{THE STATIONARY MEASURE}

Our goal in this section is to derive an explicit formula for the probabilities of all configurations, in the stationary measure, for a system of $K_{1}$ first-class and $K_{2}$ second-class particles on a ring of $N$ sites. In an earlier study ${ }^{(19)}$ of a system with open boundaries and with only first-class particles such formulas were given in terms of certain matrix products. Here we extend this method to the case of periodic boundary conditions for a system with an arbitrary number of second-class particles.

If the system contains no second-class particles, then the stationary measure is well known: all configurations are equally likely. For the rest of the section we will therefore assume that the system contains at least one second-class particle. We will show that the stationary measure may be constructed with the aid of two matrices or operators $D$ and $E$ which satisfy ${ }^{(19)}$

$$
D E=D+E
$$

If we define

$$
A=D E-E D
$$


then it follows from (3.1) that $A$ is a projection $\left(A^{2}=A\right)$ and satisfies

$$
D A=A \quad \text { and } \quad A E=A
$$

We suppose also that trace $A<\infty$. (An example of such matrices will be given below.) Then we claim that for fixed $K=\left(K_{0}, K_{1}, K_{2}\right)$ the probability in the stationary measure of the configuration $\tau$ is given, up to an overall normalizing factor dependent on $K$, by the weight $w_{K}(\tau)$ which is defined by

$$
w_{K}\left(\tau_{1}, \ldots, \tau_{N}\right) \equiv \operatorname{trace}\left[X_{1} \cdots X_{N}\right]
$$

where $X_{i}=E$ if $\tau_{i}=0, X_{i}=D$ if $\tau_{i}=1$, and $X_{i}=A$ if $\tau_{i}=2$. Note that the invariance of the system under rotation of the ring of sites, that is, under cyclic permutation of the $\tau_{i}$, is preserved by the corresponding invariance of the trace.

We next write down the stationarity conditions on the measure which follow from the dynamics described in Section 2. In doing so we will use the following standard notation: if $\tau=\left(\tau_{1}, \ldots, \tau_{N}\right)$ is a system configuration, then $\tau^{i j}$ denotes the configuration obtained from $\tau$ by interchanging $\tau_{i}$ and $\tau_{j}$. Now consider the possible change in the probability of a specific configuration $\tau$ during some time interval $d t$. For each pair of indices $i, i+1$ with $\tau_{i} \tau_{i+1}$ of the form 10,12 , or 20 there is a probability $d t$ that the states of these sites will be interchanged during the time interval; on the other hand, for each such pair $i, i+1$ with $\tau_{i} \tau_{i+1}$ of the form 01,21 , or 02 there is a probability $d t$ that an exchange will occur in the configuration $\tau^{i, i+1}$, leading to the configuration $\tau$. Thus the condition for stationarity of the measure with weights $w_{K}$ is that for all $\tau$,

$$
\sum_{\left\{i \mid \tau_{i} \tau_{i+1}=10,12, \text { or } 20\right\}} w_{K}(\tau)=\sum_{\left\{i \mid \tau_{i} \tau_{i+1}=01,21, \text { or } 02\right\}} w_{K}\left(\tau^{i, i+1}\right)
$$

We now verify that (3.4) provides the weights of a stationary measure, that is, according to (3.5), that for all $\tau$,

$$
\begin{gathered}
\sum_{\left\{i \mid \tau_{i} \tau_{i+1}=10,12, \text { or } 20\right\}} \operatorname{trace}\left[X_{1} \cdots X_{i} X_{i+1} \cdots X_{N}\right] \\
=\sum_{\left\{i \mid \tau_{i} \tau_{i+1}=01,21, \text { or } 02\right\}} \operatorname{trace}\left[X_{1} \cdots X_{i+1} X_{i} \cdots X_{N}\right]
\end{gathered}
$$

Let us write $X_{1} \cdots X_{N}$, possibly after a cyclic permutation which will not affect the trace, in terms of blocks of consecutive identical matrices:

$$
X_{1} \cdots X_{N}=Y_{1}^{k_{1}} \ldots Y_{m}^{k_{m}}
$$

where each $Y_{j}$ is $D, A$, or $E$, and $Y_{j} \neq Y_{j+1}, Y_{m} \neq Y_{1}$. Now in each term on the left-hand side of (3.6) we use the algebraic relations (3.1) and (3.3) 
to replace the product $X_{i} X_{i+1}$ by a sum of two operators or a single operator-that is, $D E$ is replaced by $D+E$, while $D A$ and $A E$ are replaced by $A$. The net effect of these replacements is that each boundary $D E, D A$, or $A E$ between blocks in the product gives rise to one or two terms:

$$
\begin{aligned}
& \cdots D E \cdots \rightarrow \operatorname{trace}[\cdots \hat{D} E \cdots]+\operatorname{trace}[\cdots D \hat{E} \cdots] \\
& \cdots D A \cdots \rightarrow \operatorname{trace}[\cdots \hat{D} A \cdots] \\
& \cdots A E \cdots \rightarrow \operatorname{trace}[\cdots A \hat{E} \cdots]
\end{aligned}
$$

where the caret denotes an omitted factor. Since each $D$ block is followed by an $E$ or an $A$, and each $E$ block is preceded by a $D$ or an $A$, each $D$ block (respectively $E$ block) gives rise to a term in which the rightmost (respectively leftmost) factor in the block has disappeared; notice that in the case of a $D E$ boundary we get a term for each block. Thus we find

$$
\begin{aligned}
& \sum_{\left\{i \mid t_{i} \tau_{i+1}=10,12, \text { or 20 }\right\}} \operatorname{trace}\left[X_{1} \cdots X_{i} X_{i+1} \cdots X_{N}\right] \\
= & \sum_{\left\{j \mid Y_{j}=D, E\right\}} \operatorname{trace}\left[Y_{1}^{k_{1}} \cdots Y_{j}^{k_{j}-1} \cdots Y_{m}^{k_{m}}\right]
\end{aligned}
$$

Similarly, each block boundary $X_{i} X_{i+1}$ of the form $E D, A D$, or $E A$ contributes a term to the right-hand side of (3.6) in which $X_{i} X_{i+1}$ has become $X_{i+1} X_{i}$; if we make the same replacements as above for the latter product, then the net effect on block boundaries is

$$
\begin{aligned}
& \cdots E D \cdots \rightarrow \cdots D E \cdots \rightarrow \operatorname{trace}[\cdots E \hat{D} \cdots]+\operatorname{trace}[\cdots \hat{E} D \cdots] \\
& \cdots A D \cdots \rightarrow \cdots D A \cdots \rightarrow \operatorname{trace}[\cdots A \hat{D} \cdots] \\
& \cdots E A \cdots \rightarrow \cdots A E \cdots \rightarrow \operatorname{trace}[\cdots \hat{E} A \cdots]
\end{aligned}
$$

Now the $D$ blocks lose their leftmost and the $E$ blocks their rightmost factors, and we find that

$$
\begin{aligned}
& \left.\sum_{\left\{i \mid \tau_{i} \tau_{i+1}\right.}=01,21, \text { or } 02\right\} \\
= & \sum_{\left\{j \mid Y_{j}=D, E\right\}} \operatorname{trace}\left[X_{1} \cdots X_{i+1} X_{i} \cdots X_{N}\right]
\end{aligned}
$$

Comparison of (3.8) and (3.10) verifies (3.6).

We turn finally to the question of existence of matrices $D$ and $E$ satisfying (3.1). If we write $D=I+d$ and $E=I+e$, then the algebra (3.1) satisfied by $D$ and $E$ reduces to $d e=I$, so that if $D$ and $E$ are finite dimensional, then $d=e^{-1}, D$ and $E$ commute, and $A=0$. This trivial solution may in fact be regarded as giving the correct weights for the system with 
no second-class particles. To treat second-class particles, however, we must take $D$ and $E$ to be infinite dimensional; a simple solution ${ }^{(19)}$ of $d e=I$ with noncommuting $d, e$ takes $e$ to be the right shift operator on semi-infinite vectors and $d$ to be its adjoint, leading to

$$
D=\left(\begin{array}{ccccc}
1 & 1 & 0 & 0 & \cdots \\
0 & 1 & 1 & 0 & \\
0 & 0 & 1 & 1 & \\
0 & 0 & 0 & 1 & \\
\vdots & & & & \ddots
\end{array}\right), \quad E=\left(\begin{array}{ccccc}
1 & 0 & 0 & 0 & \ldots \\
1 & 1 & 0 & 0 & \\
0 & 1 & 1 & 0 & \\
0 & 0 & 1 & 1 & \\
\vdots & & & \ddots & \ddots
\end{array}\right)
$$

$A$ is then the projection on the first component of the vector and has trace 1:

$$
A=|1\rangle\langle 1|=\left(\begin{array}{ccccc}
1 & 0 & 0 & 0 & \cdots \\
0 & 0 & 0 & 0 & \\
0 & 0 & 0 & 0 & \\
0 & 0 & 0 & 0 & \\
\vdots & & \ddots & \ddots
\end{array}\right)
$$

where $\langle 1|=[1,0,0, \ldots]$. Of course, our results are independent of any explicit representation of the matrices, depending only on (3.1), (3.2), and the fact that $A$ has finite trace. For notational convenience in the balance of the paper, however, we will assume that $A$ is a one-dimensional projector and write $A=|1\rangle\langle 1|$ as in (3.12). We will also use the specific representation (3.11), (3.12) in the Appendix.

There is, however, one important property of the stationary state which is easy to see in this representation. Let us consider configurations $\left(\tau_{1}, \ldots, \tau_{N}\right)$ which have second-class particles at two specified sites, say $j$ and $N$. Then the weights of such configurations factorize:

$$
w_{K}\left(\tau_{1}, \ldots, \tau_{N}\right)=\left\langle 1\left|X_{1} \cdots X_{j-1}\right| 1\right\rangle\left\langle 1\left|X_{j+1} \cdots X_{N-1}\right| 1\right\rangle
$$

when $\tau_{j}=\tau_{N}=2$. In later sections we will consider the infinite-volume limit of these systems, conditioning on the presence of a second-class particle at site $N$, or equivalently at the origin in the limit. Because of the factorization (3.13), there is in the limit independence between the portions of the system to the left and right of the origin, as expressed by (2.3).

In the remainder of the paper we will consider several different infinitevolume limits of the system described here, in which we either fix a finite number of second-class particles or consider a finite density of second-class particles. In each case we will concentrate on calculating the limiting values 
of the particle density profiles; however, it is easy to extend the computations to show that the (weak) limit of the measure defined here-that is, the limit of all correlation functions-exists in each case.

\section{THE PROFILE SEEN FROM A SINGLE SECOND-CLASS PARTICLE}

In this section we consider systems which contain a single second-class particle, and ultimately derive an exact expression for the stationary density profile of first-class particles, seen from the second-class particle, in an infinite system in which the first-class particles have average density $\rho$. In the process we also find formulas for this profile in the canonical and grand canonical ensembles for finite systems.

We begin by considering a system of $N$ sites, with a single second-class particle $\left(K_{2}=1\right)$ and $K_{1}=K$ first-class particles. Since the cyclic symmetry of the model implies that the weights of configurations in the steady state depend only on the positions of the first-class particles relative to the second-class particle, we may choose a frame in which the second-class particle is always at position $N$. Equation (3.4), with the notation $A=|1\rangle\langle 1|$, then becomes

$$
w_{K}\left(\tau_{1}, \tau_{2}, \ldots, \tau_{N-1}, 2\right)=\left\langle 1\left|\prod_{i=1}^{N-1} X_{i}\right| 1\right\rangle
$$

where $X_{i}=D$ if $\tau_{i}=1$ and $X_{i}=E$ if $\tau_{i}=0$. From this one can in principle calculate the conditional density $\left\langle\tau_{i} \mid \tau_{N}=2\right\rangle_{K}$ at any site $i \neq N$ in the system-that is, the probability that site $i$ is occupied-by

$$
\left\langle\tau_{i} \mid \tau_{N}=2\right\rangle_{K}=\frac{\sum_{\text {configurations }} w_{K}\left(\tau_{1}, \tau_{2}, \ldots, \tau_{N-1}, 2\right) \tau_{i}}{\sum_{\text {configurations }} w_{K}\left(\tau_{1}, \tau_{2}, \ldots, \tau_{N-1}, 2\right)}
$$

where the sums run over all the configurations with $K$ first-class particles and $K_{0}=N-1-K$ empty sites.

The quantities $\left\langle\tau_{i} \mid \tau_{N}=2\right\rangle_{K}$ can in fact be computed from (4.2) (see Remark 4.1 at the end of this section), but the constraint of a fixed number of first-class particles makes the calculation rather complicated. It is more convenient to work in the grand canonical ensemble, in which the number of first-class particles can fluctuate. For this purpose we introduce two parameters which play the role of fugacities: $x$ for first-class particles and $z$ for empty sites. (It would be sufficient to introduce only one of these, since the total number of available sites is fixed at $N-1$, but the use of both makes the symmetry between first-class particles and holes more apparent). 
In the grand canonical ensemble the unnormalized weight $W_{N}\left(\tau_{1}, \tau_{2}, \ldots, \tau_{N-1}, 2\right)$ of the configuration $\left(\tau_{1}, \ldots, \tau_{N-1}, 2\right)$ with $K_{1}$ firstclass and $K_{0}=N-K_{1}-1$ holes is given by

$$
\begin{aligned}
W_{N}\left(\tau_{1}, \tau_{2}, \ldots, \tau_{N-1}, 2\right) & =x^{K_{1}} z^{K_{0}} w_{K}\left(\tau_{1}, \tau_{2}, \ldots, \tau_{N-1}, 2\right) \\
& =\left\langle 1\left|\prod_{i=1}^{N-1}\left[x D \tau_{i}+z E\left(1-\tau_{i}\right)\right]\right| 1\right\rangle
\end{aligned}
$$

The density $d_{i}(N)$ at site $i \neq N$ is then

$$
d_{i}(N) \equiv \frac{\sum_{\tau} W_{N}\left(\tau_{1}, \tau_{2}, \ldots, \tau_{N-1}, 2\right) \tau_{i}}{\sum_{\tau} W_{N}\left(\tau_{1}, \tau_{2}, \ldots, \tau_{N-1}, 2\right)}
$$

where the sums, no longer restricted to fixed $K_{0}$ and $K_{1}$, run over $\tau_{j}=0,1$ for $j=1, \ldots, N-1$. Introducing

$$
C=x D+z E
$$

we see that these densities are easily expressed in terms of the matrix $C$ :

$$
d_{i}(N)=x \frac{\left\langle 1\left|C^{i-1} D C^{N-i-1}\right| 1\right\rangle}{\left\langle 1\left|C^{N-1}\right| 1\right\rangle}
$$

From (3.2) and (3.3) it follows that $D|1\rangle=|1\rangle,\langle 1| E=\langle 1|$, and $D C-C D=z|1\rangle\langle 1|$, so that

$$
\begin{gathered}
d_{1}(N)=1-z \frac{\left\langle 1\left|C^{N-2}\right| 1\right\rangle}{\left\langle 1\left|C^{N-1}\right| 1\right\rangle} \\
d_{i}(N)-d_{i+1}(N)=x z \frac{\left\langle 1\left|C^{i-1}\right| 1\right\rangle\left\langle 1\left|C^{N-i-2}\right| 1\right\rangle}{\left\langle 1\left|C^{N-1}\right| 1\right\rangle}
\end{gathered}
$$

and

$$
d_{N-1}(N)=x \frac{\left\langle 1\left|C^{N-2}\right| 1\right\rangle}{\left\langle 1\left|C^{N-1}\right| 1\right\rangle}
$$

Thus the computation of the $d_{i}(N)$ is reduced to the evaluation of the matrix elements $\left\langle 1\left|C^{n}\right| 1\right\rangle$. These are calculated in the Appendix, both exactly [see (A.5)],

$$
\left\langle 1\left|C^{n}\right| 1\right\rangle=\sum_{p=0}^{n} x^{p} z^{n-p} \frac{1}{p+1}\left(\begin{array}{c}
n+1 \\
p
\end{array}\right)\left(\begin{array}{l}
n \\
p
\end{array}\right)
$$

and in the large- $n$ limit [see (A.6)],

$$
\left\langle 1\left|C^{n}\right| 1\right\rangle \simeq \frac{(\sqrt{x}+\sqrt{z})^{2 n+3}}{2 \sqrt{\pi} n^{3 / 2}(x z)^{3 / 4}}
$$

Note that these results are symmetric in $x$ and $z$. 
As always in the grand canonical ensemble approach, one must choose the values of the fugacities $x$ and $z$ in order to fix the density of the first-class particles to be $\rho$. It follows from (4.3) and (4.6) that the average number $\left\langle K_{1}\right\rangle$ of first-class particles in a system of $N$ sites (including the second-class particle, i.e., with $N-1$ sites available for the first-class particles) is

$$
\left\langle K_{1}\right\rangle=x \frac{d}{d x} \log \left\langle 1\left|C^{N-1}\right| 1\right\rangle
$$

Then for large $N$, from (4.11),

$$
\left\langle K_{1}\right\rangle \simeq N \frac{\sqrt{x}}{\sqrt{x}+\sqrt{z}}
$$

so that, in the infinite-volume limit, a density $\rho$ of first-class particles in the system is obtained when $x$ and $z$ satisfy

$$
\frac{\sqrt{x}}{\sqrt{x}+\sqrt{z}}=\rho
$$

Note that $x$ and $z$ are determined only up to an overall factor. Note also that the fluctuations in the density go to zero in this limit: from $\left\langle K_{1}^{2}\right\rangle-\left\langle K_{1}\right\rangle^{2}=x(d / d x)\left\langle K_{1}\right\rangle$ we have that for large $N$,

$$
\left\langle\left(\frac{K_{1}}{N}\right)^{2}-\left(\frac{\left\langle K_{1}\right\rangle}{N}\right)^{2}\right\rangle^{1 / 2} \simeq \frac{1}{\sqrt{N}}
$$

so that the results of the canonical and grand canonical ensembles agree in the large- $N$ limit.

We now turn to the evaluation of the infinite-volume limit of the profile. Let us define, for all $i>0, d_{i}=\lim _{N \rightarrow \infty} d_{i}(N)$ and $d_{-i}=$ $\lim _{N \rightarrow \infty} d_{N-i}(N)$. We may use the asymptotic formula (4.11) for $\left\langle 1\left|C^{n}\right| 1\right\rangle$ to evaluate the $N \rightarrow \infty$ limits of (4.9) and (4.8):

$$
d_{-1}=\frac{x}{(\sqrt{x}+\sqrt{z})^{2}}=\rho^{2}
$$

and for all $i>0$, from the exact expression (4.10) for the matrix elements of $C$,

$$
\begin{aligned}
d_{-i-1}-d_{-i} & =x z\left\langle 1\left|C^{i-1}\right| 1\right\rangle(\sqrt{x}+\sqrt{z})^{-2 i-2} \\
& =\sum_{p=0}^{i-1} \frac{1}{p+1}\left(\begin{array}{c}
i \\
p
\end{array}\right)\left(\begin{array}{c}
i-1 \\
p
\end{array}\right)(1-\rho)^{2 i-2 p} \rho^{2 p+2}
\end{aligned}
$$


These formulas determine the profile to the left of the second-class particle in the infinite-volume limit: for $i>0$,

$$
d_{-i}=\rho^{2}+\sum_{n=1}^{i-1} \sum_{p=0}^{n-1} \frac{1}{p+1}\left(\begin{array}{c}
n \\
p
\end{array}\right)\left(\begin{array}{c}
n-1 \\
p
\end{array}\right)(1-\rho)^{2 n-2 p} \rho^{2 p+2}
$$

Similarly, the $N \rightarrow \infty$ limits of (4.7) and (4.8) determine the profile to the right of the second-class particle:

$$
d_{1}=1-\frac{z}{(\sqrt{x}+\sqrt{z})^{2}}=2 \rho-\rho^{2}
$$

and for $i>0$,

$$
d_{i}-d_{i+1}=x z\left\langle 1\left|C^{i-1}\right| 1\right\rangle(\sqrt{x}+\sqrt{z})^{-2 i-2}=d_{-i-1}-d_{-i}
$$

Then

$$
d_{i}=2 \rho-\rho^{2}-\sum_{n=1}^{i-1} \sum_{p=0}^{n-1} \frac{1}{p+1}\left(\begin{array}{l}
n \\
p
\end{array}\right)\left(\begin{array}{c}
n-1 \\
p
\end{array}\right)(1-\rho)^{2 n-2 p} \rho^{2 p+2}
$$

For example, considering (4.18) and (4.21) for $i=2$ and 3 yields

$$
\begin{aligned}
d_{-2} & =2 \rho^{2}-2 \rho^{3}+\rho^{4}, & d_{-3} & =3 \rho^{2}-6 \rho^{3}+9 \rho^{4}-6 \rho^{5}+2 \rho^{6} \\
d_{2} & =2 \rho-2 \rho^{2}+2 \rho^{3}-\rho^{4}, & d_{3} & =2 \rho-3 \rho^{2}+6 \rho^{3}-9 \rho^{4}+6 \rho^{5}-2 \rho^{6}
\end{aligned}
$$

and so on.

With the aid of the formula [see (A.9)]

$$
\sum_{i=1}^{\infty}\left\langle 1\left|C^{i-1}\right| 1\right\rangle(\sqrt{x}+\sqrt{z})^{-2 i+2}=\frac{1}{\rho(1-\rho)}
$$

we see that

$$
\lim _{i \rightarrow \infty} d_{-i}=d_{-1}+x z \sum_{i=1}^{\infty}\left\langle 1\left|C^{i-1}\right| 1\right\rangle(\sqrt{x}+\sqrt{z})^{-2 i-2}=\rho
$$

This is of course expected, since at large distance we should recover the uniform density $\rho$. Similarly, $\lim _{i \rightarrow \infty} d_{i}=\rho$. We may also calculate the approach to this limiting density:

$$
d_{-i-1}-d_{-i} \simeq \frac{x z}{2 \sqrt{\pi}(\sqrt{x}+\sqrt{z})(x z)^{3 / 4}} \frac{1}{i^{3 / 2}}=\left[\frac{\rho(1-\rho)}{4 \pi}\right]^{1 / 2} \frac{1}{i^{3 / 2}}
$$


for large $i$, which implies that

$$
\rho-d_{-i} \simeq\left[\frac{\rho(1-\rho)}{\pi}\right]^{1 / 2} \frac{1}{i^{1 / 2}}
$$

We see that the profile decays as the square root of the distance; the mean field theory ${ }^{(16)}$ predicts an exponent 1.

Note that from (4.18) and (4.21) we recover that fact that $d_{i}+d_{-i}=2 \rho$ for all $i$, as already known from (2.2).

Remark 4.1. The results obtained within the grand canonical ensemble can be recovered from a direct calculation in the canonical ensemble with $K$ first-class particles, that is, from (4.2). For this purpose we consider the denominator $Z_{N}(K)$ of $(4.2)$ :

$$
Z_{N}(K) \equiv \sum_{\text {configurations }} w_{K}\left(\tau_{1}, \tau_{2}, \ldots, \tau_{N-1}, 2\right)
$$

where the sum is restricted to the configurations with one second-class particle on site $N$ and $K$ first-class particles distributed on the remaining $N-1$ sites. If we multiply $Z_{N}(K)$ by $x^{K} z^{N-1-K}$ and sum over $K$, we obtain [see (4.3)] the grand canonical partition function:

$$
\sum_{K=0}^{N-1} x^{K} z^{N-1-K} Z_{N}(K)=\left\langle 1\left|C^{N-1}\right| 1\right\rangle
$$

If we now compare the coefficients of $x$ and $z$ in (4.10) and (4.29), we see that

$$
Z_{N}(K)=\frac{1}{K+1}\left(\begin{array}{c}
N \\
K
\end{array}\right)\left(\begin{array}{c}
N-1 \\
K
\end{array}\right)
$$

We can then make use of the commutation relations and some algebra to find formulas for the average occupations in this ensemble. For example,

$$
\begin{aligned}
\left\langle\tau_{N-1}\right\rangle_{K} & =\frac{Z_{N-1}(K-1)}{Z_{N}(K)}=\frac{K(K+1)}{N(N-1)} \\
\left\langle\tau_{N-2}\right\rangle_{K} & =\frac{Z_{N-1}(K-1)+Z_{N-2}(K-1)}{Z_{N}(K)} \\
& =\frac{K(K+1)}{N(N-1)}+\frac{K(K+1)(N-K)(N-K-1)}{N(N-1)^{2}(N-2)}
\end{aligned}
$$

and so on. In the limit $K, N \rightarrow \infty$, with $K / N \rightarrow \rho$, we find that $\left\langle\tau_{N-i}\right\rangle_{K}$ converges to $d_{-i}$; for example, (4.31) and (4.32) recover (4.16) and (4.22). 


\section{THE BOUND STATE BETWEEN TWO SECOND-CLASS PARTICLES}

In this section we consider the case of two second-class particles in a system of first-class particles at density $\rho$. We will show that in the infinitevolume limit the two second-class particles form a bound state, and that the probability of finding them a distance $r$ apart has power-law behavior, decaying as $r^{-3 / 2}$ for large $r$.

From our formula (3.4) for the weights $w_{k}$ as traces we know that in the canonical ensemble, with two second-class particles and a fixed number $K=K_{1}$ of first-class particles, the weight for the configuration with the second-class particles $r$ units apart [with $1 \leqslant r \leqslant(N-1) / 2$ ] -say at positions $N$ and $r$-is easily computable if we specify the occupation variables for the other sites to be $\tau_{i}=0,1$. This weight (if we take $N$ odd to simplify counting) is given by

$w_{K}\left(\tau_{1}, \ldots, \tau_{r-1}, 2, \tau_{r+1}, \ldots, \tau_{N-1}, 2\right)=\left\langle 1\left|\prod_{i=1}^{r-1} X_{i}\right| 1\right\rangle\left\langle 1\left|\prod_{i=r+1}^{N-1} X_{i}\right| 1\right\rangle$

where $X_{i}=D$ if $\tau_{i}=1$ and $X_{i}=E$ if $\tau_{i}=0$. As in the previous section, it is convenient to introduce the grand canonical ensemble with fugacities $x$ for first-class particles and $z$ for holes. Then from (5.1) the probability $p_{N}(r)$ of finding the two second-class particles a distance $r$ apart is

$$
\begin{aligned}
p_{N}(r)= & \left\langle 1\left|C^{r-1}\right| 1\right\rangle\left\langle 1\left|C^{N-r-1}\right| 1\right\rangle \\
& \times\left[\sum_{s=1}^{(N-1) / 2}\left\langle 1\left|C^{s-1}\right| 1\right\rangle\left\langle 1\left|C^{N-s-1}\right| 1\right\rangle\right]^{-1}
\end{aligned}
$$

where $C=x D+z E$ as above. In the infinite-volume limit we must again choose $x$ and $z$ to satisfy (4.14) to ensure that the density of first-class particles is $\rho$; then the probability $p_{\infty}(r)=\lim _{N \rightarrow \infty} p_{N}(r)$ that the particles are a distance $r$ apart is

$$
\begin{aligned}
p_{\infty}(r) & =\left\langle 1\left|C^{r-1}\right| 1\right\rangle(\sqrt{x}+\sqrt{z})^{-2(r-1)}\left[\sum_{s=0}^{\infty}\left\langle 1\left|C^{s}\right| 1\right\rangle(\sqrt{x}+\sqrt{z})^{-2 s}\right]^{-1} \\
& =\rho(1-\rho) \sum_{p=0}^{r-1} \rho^{2 p}(1-\rho)^{2 r-2 p-2} \frac{1}{p+1}\left(\begin{array}{c}
r \\
p
\end{array}\right)\left(\begin{array}{c}
r-1 \\
p
\end{array}\right)
\end{aligned}
$$

For small $r$ we may use (5.4) to find $p_{\infty}(r)$ explicitly; for example, $p_{\infty}(1)=\rho(1-\rho)$ and $p_{\infty}(2)=\rho(1-\rho)\left[\rho^{2}+(1-\rho)^{2}\right]$. For large $r$ we may either estimate $p_{\infty}(r)$ directly from (5.4) or use (5.3) together with the 
asymptotic results (4.24) and (4.11) for the matrix elements of $C$; in either case, we find

$$
p_{\infty}(r) \simeq \frac{1}{2[\pi \rho(1-\rho)]^{1 / 2}} \frac{1}{r^{3 / 2}}
$$

Thus the two second-class particles form a rather unusual bound state: the decay of $p_{\infty}(r)$ is a power law and the average distance between the two second-class particles is infinite.

It is easy to extend this analysis to a finite system with any fixed number $m$ of second-class particles. We find that in the limit $N \rightarrow \infty$ the $m$ particles form a bound state in which the probability for relative distances $r_{i}$ between the $i$ th and the $(i+1)$ th particles is $\prod_{i=1}^{m-1} p_{\infty}\left(r_{i}\right)$. This factorization is an example of the decoupling of the system across second-class particles discussed at the end of Section 3.

Remark 5.1. All these results can be easily recovered with the canonical ensemble. Here

$$
\begin{aligned}
p_{N}(r)= & \sum_{k=0}^{r-1} Z_{r}(k) Z_{N-r}(K-k) \\
& \times\left[\sum_{s=1}^{(N-1) / 2} \sum_{q=0}^{s-1} Z_{s}(q) Z_{N-s}(K-q)\right]^{-1}
\end{aligned}
$$

where $Z_{N}(k)$ is given by (4.30). Now one can show that if $N, K \rightarrow \infty$ with $K / N \rightarrow \rho$ (and $r$ and $k$ are fixed),

$$
Z_{N-r}(K-k) / Z_{N}(K) \simeq \rho^{2 k}(1-\rho)^{2(r-k)}
$$

so that (5.6) becomes

$$
\begin{aligned}
p_{\infty}(r)= & \sum_{k=0}^{r-1} Z_{r}(k) \rho^{2 k}(1-\rho)^{2(r-k-1)} \\
& \times\left[\sum_{s=1}^{\infty} \sum_{q=0}^{s-1} Z_{s}(q) \rho^{2 q}(1-\rho)^{2(s-q-1)}\right]^{-1}
\end{aligned}
$$

The denominator in (5.7) is, from (4.29) and (4.24),

$$
\sum_{N=0}^{\infty}\left\langle 1\left|C^{N-1}\right| 1\right\rangle(\sqrt{x}+\sqrt{z})^{-2(N-1)}=\frac{1}{\rho(1-\rho)}
$$

and therefore with (4.30) one recovers (5.4). We note that the argument in fact gives additional information: the probability that the two second-class 
particles are a distance $r$ apart with exactly $k$ first-class particles between them. In the infinite system this probability is

$Z_{r}(k)(1-\rho)^{2 r-2 k-1} \rho^{2 k+1}=\frac{1}{k+1}\left(\begin{array}{l}r \\ k\end{array}\right)\left(\begin{array}{c}r-1 \\ k\end{array}\right)(1-\rho)^{2 r-2 k-1} \rho^{2 k+1}$

\section{A POSITIVE DENSITY OF SECOND-CLASS PARTICLES}

In this section we study the infinite-volume limit in the case in which the first- and second-class particles have positive densities $\rho_{1}$ and $\rho_{2}$, respectively. We begin with a finite system-a ring of $N$ sites-and since we want to calculate the particle densities as seen from a second-class particle, we choose the frame in which site $N$ is occupied by such a particle. One approach is to work in the canonical ensemble, with $K_{1}$ first-class and $K_{2}$ second-class particles, and then take the limit $N, K_{1}, K_{2} \rightarrow \infty$ with $K_{1} / N \rightarrow \rho_{1}$ and $K_{2} / N \rightarrow \rho_{2}$. As in the previous sections, however, it is more convenient to work in the grand canonical ensemble for the sites $1, \ldots, N-1$.

We therefore introduce three fugacities (two would be sufficient), $x$ for the first-class particles, $y$ for the second-class particles, and $z$ for the holes, and define a matrix $G$ as the obvious generalization of the matrix $C$ introduced previously:

$$
G=x D+y A+z E
$$

Then the densities $d_{i}(N), a_{i}(N)$, and $e_{i}(N)$ of first-class particles, secondclass particles, and holes, respectively, are given by

$$
\begin{aligned}
& d_{i}(N)=x \frac{\left\langle 1\left|G^{i-1} D G^{N-i-1}\right| 1\right\rangle}{\left\langle 1\left|G^{N-1}\right| 1\right\rangle} \\
& a_{i}(N)=y \frac{\left\langle 1\left|G^{i-1} A G^{N-i-1}\right| 1\right\rangle}{\left\langle 1\left|G^{N-1}\right| 1\right\rangle}
\end{aligned}
$$

and

$$
e_{i}(N)=z \frac{\left\langle 1\left|G^{i-1} E G^{N-i-1}\right| 1\right\rangle}{\left\langle 1\left|G^{N-1}\right| 1\right\rangle}
$$

In the Appendix we give a recursion relation (A.12) and from that an explicit formula [see (A.14)] for the matrix elements $\left\langle 1\left|G^{n}\right| 1\right\rangle$; to complete the determination of the densities, we will express the numerators in (6.2)-(6.4) in terms of the known quantities $\left\langle 1\left|G^{n}\right| 1\right\rangle$ and $\left\langle 1\left|C^{n}\right| 1\right\rangle$. 
First, it is simple to obtain the explicit form for the density of secondclass particles: since $A=|1\rangle\langle 1|$, we have from (6.3) that

$$
a_{i}(N)=y \frac{\left\langle 1\left|G^{i-1}\right| 1\right\rangle\left\langle 1\left|G^{N-i-1}\right| 1\right\rangle}{\left\langle 1\left|G^{N-1}\right| 1\right\rangle}
$$

To treat the density $d_{i}(N)$ of first-class particles we first note that, from (6.2) and the fact that $D|1\rangle=|1\rangle$ [see (3.3)],

$$
d_{N-1}(N)=x \frac{\left\langle 1\left|G^{N-2}\right| 1\right\rangle}{\left\langle 1\left|G^{N-1}\right| 1\right\rangle}
$$

Next we note a simple consequence of the matrix algebra (3.1)-(3.3):

$$
\begin{aligned}
x(D G-G D) & =x y(D A-A D)+x z(D E-E D) \\
& =(x y+x z) A-x y A D=(x+y)(z+y) A-y A G
\end{aligned}
$$

Clearly (6.2) and (6.7) imply that for $i>1$,

$$
\begin{aligned}
& d_{i}(N)-d_{i+1}(N)= \\
& \frac{(x+y)(z+y)\left\langle 1\left|G^{i-1}\right| 1\right\rangle\left\langle 1\left|G^{N-i-2}\right| 1\right\rangle-y\left\langle 1\left|G^{i-1}\right| 1\right\rangle\left\langle 1\left|G^{N-i-1}\right| 1\right\rangle}{\left\langle 1\left|G^{N-1}\right| 1\right\rangle}
\end{aligned}
$$

which can be rewritten using the recursion (A.12) as

$$
d_{i}(N)-d_{i+1}(N)=x z \frac{\left\langle 1\left|G^{i-1}\right| 1\right\rangle\left\langle 1\left|C^{N-i-2}\right| 1\right\rangle}{\left\langle 1\left|G^{N-1}\right| 1\right\rangle}
$$

The formulas (6.6) and (6.9) determine all the $d_{i}(N)$.

The determination of the $e_{i}(N)$ is similar. First,

$$
e_{1}(N)=z \frac{\left\langle 1\left|G^{N-2}\right| 1\right\rangle}{\left\langle 1\left|G^{N-1}\right| 1\right\rangle}
$$

Then, from

$$
\begin{aligned}
z(E G-G E) & =z x(E D-D E)+z y(E A-A E) \\
& =-(z x+z y) A+z y E A=-(x+y)(z+y) A+y G A
\end{aligned}
$$

and (A.12) we find

$$
e_{i}(N)-e_{i+1}(N)=-x z \frac{\left\langle 1\left|C^{i-1}\right| 1\right\rangle\left\langle 1\left|G^{N-i-2}\right| 1\right\rangle}{\left\langle 1\left|G^{N-1}\right| 1\right\rangle}
$$

Equations (6.10) and (6.12) determine all the $e_{i}(N)$. 
We now consider the infinite-volume limit of these densities. The large- $n$ behavior of $\left\langle 1\left|G^{n}\right| 1\right\rangle$ for $\left.y^{2}\right\rangle x z$ is determined in (A.16):

$$
\left\langle 1\left|G^{n}\right| 1\right\rangle \simeq\left(1-\frac{x z}{y^{2}}\right)\left[\frac{(y+x)(y+z)}{y}\right]^{n}
$$

From this we may determine the values of $x, y$, and $z$ which in the large- $N$ limit give densities $\rho_{1}$ of first-class particles, $\rho_{2}$ of second-class particles, and $1-\rho_{1}-\rho_{2}$ of holes:

$$
\begin{aligned}
\rho_{1} & =\lim _{N \rightarrow \infty} \frac{x}{N} \frac{d}{d x} \log \left\langle 1\left|G^{N}\right| 1\right\rangle=\frac{x}{y+x} \\
\rho_{2} & =\lim _{N \rightarrow \infty} \frac{y}{N} \frac{d}{d y} \log \left\langle 1\left|G^{N}\right| 1\right\rangle=\frac{y^{2}-x z}{(y+x)(y+z)} \\
1-\rho_{1}-\rho_{2} & =\lim _{N \rightarrow \infty} \frac{z}{N} \frac{d}{d z} \log \left\langle 1\left|G^{N}\right| 1\right\rangle=\frac{z}{y+z}
\end{aligned}
$$

It is clear from (6.15) that the assumption $y^{2}>x z$ leads to a consistent determination of $x, y$, and $z$, unique up to an overall factor; for $y \searrow(x z)^{1 / 2}$ we recover $\rho_{1}=\sqrt{x} /(\sqrt{x}+\sqrt{z})$ as in the case (4.14) with a finite number of second-class particles. (For $y^{2}<x z$ a calculation similar to that yielding (A.16) shows that $\left\langle 1\left|G^{n}\right| 1\right\rangle \simeq\left[1-y /(x z)^{1 / 2}\right]^{-2}\left\langle 1\left|C^{n}\right| 1\right\rangle$, so that the number of second-class particles in the infinite system is finite and diverges when $\left.y \nearrow(x z)^{1 / 2}\right)$.

We can now compute the infinite-volume limit of the densities $d_{i}(N)$, $a_{i}(N)$, and $e_{i}(N)$ at site $i$ relative to the second-class particle; as in Section 3, we define $d_{i}=\lim _{N \rightarrow \infty} d_{i}(N)$ and $d_{-i}=\lim _{N \rightarrow \infty} d_{N-i}(N)$, for $i>0$, with similar definitions for $a_{ \pm i}$ and $e_{ \pm i}$. We begin with a discussion of the situation to the left of the origin. From (6.6), (6.13), and (6.14)-(6.15),

$$
d_{-1}=\frac{x y}{(y+x)(y+z)}=\rho_{1}\left(\rho_{1}+\rho_{2}\right)
$$

while the difference equation (6.9) yields

$$
\begin{aligned}
d_{-i}= & d_{-1}+x z \sum_{n=1}^{i-1}\left(\frac{y}{(y+x)(y+z)}\right)^{n+1}\left\langle 1\left|C^{n-1}\right| 1\right\rangle \\
= & \rho_{1}\left(\rho_{1}+\rho_{2}\right) \\
& +\sum_{n=1}^{i-1} \sum_{p=0}^{n-1} \frac{1}{p+1}\left(\begin{array}{c}
n \\
p
\end{array}\right)\left(\begin{array}{c}
n-1 \\
p
\end{array}\right) \\
& \times\left[\rho_{1}\left(\rho_{1}+\rho_{2}\right)\right]^{p+1}\left[\left(1-\rho_{1}\right)\left(1-\rho_{1}-\rho_{2}\right)\right]^{n-p}
\end{aligned}
$$


where we have used the asymptotic behavior (4.10) of $\left\langle 1\left|C^{n}\right| 1\right\rangle$ in the last step. Moreover, because the condition $y^{2}>x z$ guarantees that the asymptotic growth of $\left\langle 1\left|C^{n}\right| 1\right\rangle$ is slower than that of $\left\langle 1\left|G^{n}\right| 1\right\rangle$ [compare (6.13) and (4.11)], the difference equation (6.12) for the $e_{i}$ implies that $e_{-i}=e_{-(i+1)}=e_{-1}$ for all $i>0$. This constant density is easily determined from $e_{-1}=1-a_{-1}-d_{-1}$; since (6.5) and (6.13) imply that

$$
a_{-1}=y^{2} /(y+x)(y+z)=\rho_{2}+\rho_{1}\left(1-\rho_{1}-\rho_{2}\right)
$$

using the value (6.17) for $d_{-1}$ yields

$$
e_{-i}=e_{-1}=1-a_{-1}-d_{-1}=1-\rho_{1}-\rho_{2}
$$

Finally, the density of second-class particles is determined simply from the above expressions for $d_{-i}$ and $e_{-i}$ :

$$
a_{-i}=1-e_{-i}-d_{-i}=\rho_{1}+\rho_{2}-d_{-i}
$$

We obtain in a very similar way expressions for the densities to the right of the second-class particle. From (6.10),

$$
e_{1}=\left(1-\rho_{1}\right)\left(1-\rho_{1}-\rho_{2}\right)
$$

and then from the difference equation (6.12), for all $i>0$,

$$
\begin{aligned}
e_{i}= & \left(1-\rho_{1}\right)\left(1-\rho_{1}-\rho_{2}\right) \\
& +\sum_{n=1}^{i-1} \sum_{p=0}^{n-1} \frac{1}{p+1}\left(\begin{array}{c}
n \\
p
\end{array}\right)\left(\begin{array}{c}
n-1 \\
p
\end{array}\right) \\
& \times\left[\rho_{1}\left(\rho_{1}+\rho_{2}\right)\right]^{p+1}\left[\left(1-\rho_{1}\right)\left(1-\rho_{1}-\rho_{2}\right)\right]^{n-p}
\end{aligned}
$$

Finally, for all $i>0$, from (6.9) (which implies $d_{i}=d_{i+1}$ ), (6.21), and the fact that $a_{1}=\rho_{2}+\rho_{1}\left(1-\rho_{1}-\rho_{2}\right)$,

$$
d_{i}=\rho_{1}
$$

and

$$
a_{i}=1-d_{i}-e_{i}=1-\rho_{1}-e_{i}
$$

In the remainder of this section we make several comments on these results; in the next we discuss their relation to shock profiles in a system consisting only of first-class particles.

Notice from these formulas that for the measure in the infinite-volume limit, conditioned on the presence of a second-class particle at the origin, the density $e_{-i}$ of holes to the left of the origin and the density $d_{i}$ of first class particles to the right of the origin (where $i>0$ in each case) are 
constant. It is easy to extend these results and show that, under this conditioning, the holes to the left and particles to the right of the origin have Bernoulli distributions with densities $1-\rho_{1}-\rho_{2}$ and $\rho_{1}$, respectively. We have no simple explanation for this extremely simple property of the invariant measure.

Next we observe that, using (A.7), it can be shown from (6.18) that

$$
\lim _{i \rightarrow \infty} d_{-i}=\rho_{1}
$$

and that the approach is exponential, since from the difference equation (6.9) and the asymptotic formulas (4.11) and (6.13) one has

$$
\begin{aligned}
d_{-i-1}-d_{-i}= & \frac{1}{2 \sqrt{\pi} i^{3 / 2}}\left[\rho_{1}\left(\rho_{1}+\rho_{2}\right)\left(1-\rho_{1}\right)\left(1-\rho_{1}-\rho_{2}\right)\right]^{1 / 4} \\
& \times\left\{\left[\rho_{1}\left(\rho_{1}+\rho_{2}\right)\right]^{1 / 2}+\left[\left(1-\rho_{1}\right)\left(1-\rho_{1}-\rho_{2}\right)\right]^{1 / 2}\right\}^{2 i+1}
\end{aligned}
$$

Similarly

$$
\lim _{i \rightarrow \infty} e_{i}=1-\rho_{1}-\rho_{2}
$$

with the same exponential approach.

If one sets $\rho_{1}=\rho$ and $\rho_{2}=0$ in (6.26), then the difference in neighboring densities reduces to (4.26) and the decay of the density to its asymptotic value is algebraic; conversely, it is interesting to notice that the only case in which the decay of $d_{-i}$ is not exponential is $\rho_{2}=0$. As $\rho_{2} \searrow 0$, the characteristic length of the exponential decay is asymptotically $4 \rho(1-\rho) \rho_{2}^{-2}$. On the other hand, according to the remark in the paragraph above, the density of first-class particles to the right of the origin here $\left(d_{i}\right.$, with $\left.i>0\right)$ is constant for all values of $\rho_{1}, \rho_{2}$, and in particular in the $\rho_{1}=\rho, \rho_{2}=0$ limit; this is in apparent disagreement with the situation [see (4.21)] when there is only one second-class particle in the system. The resolution of this paradox is that the slow decay of the densities to their asymptotic values when $\rho_{2}=0$ [see (6.26)] implies that even in this limit an infinite number of second-class particles remain in the system. These particles form a bound state, so that the second-class particle at the origin is surrounded by infinitely many others and sees a very different environment from that seen by a single second-class particle in the presence of a density $\rho$ of first-class particles. We remark that these second-class particles are distributed as in the systems with a finite number of second-class particles discussed in Section 5: the distances between consecutive second-class particles are independent and the probability that such a distance has value $r$ is $p_{\infty}(r)$ [see (5.4)]. 
Finally, by combining the explicit expressions above for $d_{i}$ and $e_{i}$ we obtain

$$
d_{-i}+a_{i}+d_{i}=2 \rho_{1}+\rho_{2}
$$

for all $i>0$, in agreement with (2.2).

\section{MICROSCOPIC SHAPES OF FRONTS}

As observed in Section 2, the results of the previous section allow us to determine the microscopic shape of the shock front between densities $\rho_{-}=\rho_{1}$ and $\rho_{+}=\rho_{1}+\rho_{2}$ in a system of first-class particles, as seen from the position of a single second-class particle which is introduced into the system to locate the shock front. To do so we define densities $\delta_{i}$ for all $i \neq 0$ by

$$
\delta_{i}= \begin{cases}d_{i} & \text { if } \quad i<0 \\ d_{i}+a_{i} & \text { if } \quad i>0\end{cases}
$$

Thus $\delta_{i}$ is the density profile of a new species of particles, consisting of all first-class particles to the left of the origin and of all first- and second-class particles to the right of the origin. Consideration of the dynamics shows that in fact the particles of this new species, together with the distinguished second-class particle located at the origin, do behave as a system of firstclass particles as seen from a single second-class particle (in the new system one ignores interchanges between particles of the new species, and ignores also the old second-class particles to the left of the origin).

Since $\lim _{i \rightarrow \pm \infty} d_{i}=\rho_{1}$ and $\lim _{i \rightarrow \pm \infty} a_{i}=\rho_{2}$, the densities $\delta_{i}$ satisfy

$$
\lim _{i \rightarrow-\infty} \delta_{i}=\rho_{-} \quad \text { and } \quad \lim _{i \rightarrow \infty} \delta_{i}=\rho_{+}
$$

so that this is indeed a system with a shock between these two densities. The exact shock profile to the left of the origin follows from (6.18): for $i>0$,

$$
\begin{aligned}
\delta_{-i}= & \rho_{-} \rho_{+}+\sum_{n=1}^{i-1} \sum_{p=0}^{n-1} \frac{1}{p+1}\left(\begin{array}{c}
n \\
p
\end{array}\right)\left(\begin{array}{c}
n-1 \\
p
\end{array}\right) \\
& \times\left(\rho_{-} \rho_{+}\right)^{p+1}\left[\left(1-\rho_{-}\right)\left(1-\rho_{+}\right)\right]^{n-p}
\end{aligned}
$$

The shock profile to the right of the origin follows from (6.22), since $\delta_{i}=1-e_{i}$ for $i>0$ : 


$$
\begin{aligned}
\delta_{i}= & \rho_{+}+\rho_{-}\left(1-\rho_{+}\right) \\
& -\sum_{n=1}^{i-1} \sum_{p=0}^{n-1} \frac{1}{p+1}\left(\begin{array}{c}
n \\
p
\end{array}\right)\left(\begin{array}{c}
n-1 \\
p
\end{array}\right) \\
& \times\left(\rho_{-} \rho_{+}\right)^{p+1}\left[\left(1-\rho_{-}\right)\left(1-\rho_{+}\right)\right]^{n-p}
\end{aligned}
$$

For example, we find

$$
\delta_{-1}=\rho_{-} \rho_{+}, \quad \delta_{-2}=\rho_{-} \rho_{+}+\rho_{-} \rho_{+}\left(1-\rho_{-}\right)\left(1-\rho_{+}\right)
$$

and so forth; for $i$ large there is exponential convergence to $\rho_{-}$,

$$
\begin{aligned}
\delta_{-i-1}-\delta_{-i}= & \frac{\left[\rho_{-} \rho_{+}\left(1-\rho_{-}\right)\left(1-\rho_{+}\right)\right]^{1 / 4}}{2 \sqrt{\pi} i^{3 / 2}} \\
& \times\left\{\left(\rho_{-} \rho_{+}\right)^{1 / 2}+\left[\left(1-\rho_{-}\right)\left(1-\rho_{+}\right)\right]^{1 / 2}\right\}^{2 i-1}
\end{aligned}
$$

with characteristic length diverging as $\left(\rho_{+}-\rho_{-}\right)^{-2}$ when $\left(\rho_{+}-\rho_{-}\right)>0$. The corresponding results for $\delta_{i}, i>0$, may be obtained from the symmetry $\delta_{i}+\delta_{-i}=\rho_{+}+\rho_{-}$.

In the limit $\rho_{1}=\rho, \rho_{2}=0$, i.e., $\rho_{-}=\rho_{+}=\rho$, these results recover the "shock" profile found in Section 4. But, as discused at the end of Section 6, the makeup of the density profiles in the two situations is quite different. In the $\rho_{2} \searrow 0$ limit, the excess density ahead of the origin is contributed by second-class particles; the density of first-class particles is constant and equal to $\rho$ in this region. Similarly, behind the origin the density of holes is constant and equal to $1-\rho$, and the lowered density of first-class particles reflects the presence of second-class particles. The density profile $\delta_{i}$ found here coincides with the profile $d_{i}$ of Section 4 , however, because a second-class particle interacts with the second-class particles ahead of it as if they were first-class particles and with those behind it as if they were holes.

\section{CONCLUSION}

In this paper we have found an exact solution of the two-species, totally asymmetric simple exclusion process, first on a ring and then in the infinite-volume limit on the lattice $\mathbb{Z}$. From this solution we have calculated exact shock profiles in a one-species TASEP as seen from the location of a single second-class particle, both for the true shock between different asymptotic densities and for the artificial "shock" seen by the second-class particle in a uniform density of first-class particles. We have also observed that two second-class particles in a uniform density of first-class particles form a weakly bound state. 
The exact solution which we have introduced in a ring of sites can be extended in two ways to models with other jump rates. First, we may relax the requirement that the process be totally asymmetric and consider the case in which particles-both first and second class-jump to the right with probability $p>1 / 2$ and to the left with probability $q=1-p$. In this case we may find the steady state with the aid of matrices $D$ and $E$ which satisfy ${ }^{(19)}$

$$
p D E-q E D=(p-q)(D+E)
$$

$A=D E-E D$ will then satisfy

$$
p D A-q A D=(p-q) A \quad \text { and } \quad p A E-q E A=(p-q) A
$$

If we assume that trace $A$ is finite, we may again take the weight $w_{K}(\tau)$ of a configuration $\tau=\left(\tau_{1}, \ldots, \tau_{N}\right)$ to be

$$
w_{K}\left(\tau_{1}, \ldots, \tau_{N}\right)=\operatorname{trace}\left[X_{1} \cdots X_{N}\right]
$$

where $X_{i}=E$ if $\tau_{i}=0, X_{i}=D$ if $\tau_{i}=1$, and $X_{i}=A$ if $\tau_{i}=2$. By a slight generalization of the proof given in Section 3 for the $p=1$ case, we may then show that the $w_{K}$ are the weights of a steady state for the dynamics. Equations (8.1) and (8.2) may be realized by the choice ${ }^{(19)}$

$$
D=\left(\begin{array}{ccccc}
1 & \alpha_{1} & 0 & 0 & \cdots \\
0 & 1 & \alpha_{2} & 0 & \\
0 & 0 & 1 & \alpha_{3} & \\
0 & 0 & 0 & 1 & \\
\vdots & & & & \ddots
\end{array}\right), \quad E=\left(\begin{array}{ccccc}
1 & 0 & 0 & 0 & \cdots \\
\alpha_{1} & 1 & 0 & 0 & \\
0 & \alpha_{2} & 1 & 0 & \\
0 & 0 & \alpha_{3} & 1 & \\
\vdots & & & \ddots & \ddots
\end{array}\right)
$$

where $\alpha_{i}$ satisfies $\alpha_{i}^{2}=1-(q / p)^{i}$. The matrix $A=D E-E D$ is then given by

$$
A=\left(\begin{array}{ccccc}
\alpha_{1} & 0 & 0 & 0 & \cdots \\
0 & \alpha_{2}-\alpha_{1} & 0 & 0 & \\
0 & 0 & \alpha_{3}-\alpha_{2} & 0 & \\
0 & 0 & 0 & \alpha_{4}-\alpha_{3} & \\
\vdots & & & \ddots
\end{array}\right)
$$

Because we have assumed that $q<p$, the matrix elements of $A$ decrease exponentially and trace $A=\lim _{i \rightarrow \infty} \alpha_{i}=1$.

A different extension is to the case in which the asymmetry is still total-only jumps to the right are permitted-but the three types of jumps, $10 \rightarrow 01,12 \rightarrow 21$, and $20 \rightarrow 02$, occur at different rates, say 
$1, r$, and $s$, respectively. Now the invariant weights are given by $(8.3)$ if the matrices $D, E$, and $A$ satisfy

$$
D E=(D+E), \quad r D A=A, \quad \text { and } \quad s A E=A
$$

The proof is as in Section 3. To satisfy (8.6) we may, as in the case $r=s=1$ considered in the body of the paper, take $A$ to be a projection operator, ${ }^{(19)}$ but now $A=|V\rangle\langle W|$, with $|V\rangle$ a right eigenveetor of $D$ and $\langle W|$ a left eigenvector of $E: D|V\rangle=r^{-1}|V\rangle$ and $\langle W| E=s^{-1}\langle W|$. Several explicit realizations of such $D, E,|V\rangle$, and $\langle W|$ are given in ref. 19 .

\section{APPENDIX}

In this Appendix we collect various formulas involving the matrix elements of powers of the fundamental matrices $C$ and $G$ used in computations in the grand canonical ensembles.

We begin with the calculation of $\left\langle 1\left|C^{n}\right| 1\right\rangle$. The matrix $C=x D+z E$ defined in (4.5) is a tridiagonal matrix in the representation defined in (3.11):

$$
C=\left(\begin{array}{ccccc}
x+z & x & 0 & 0 & \cdots \\
z & x+z & x & 0 & \\
0 & z & x+z & x & \\
0 & 0 & z & x+z & \\
\vdots & & & \ddots & \ddots
\end{array}\right)
$$

We may think of $C$ as the unnormalized transition matrix of a one-dimensional walk in which the walker can at each step go to the right with a weight $x$, go to the left with a weight $z$, or remain at its present position with a weight $x+z$; because the matrix is semi-infinite, the walk cannot go to the left of site 1 . The quantity $\left\langle 1\left|C^{n}\right| 1\right\rangle$ is thus just the total weight of all the walks of $n$ steps starting and ending at 1 . But the number of such walks which take a total of $p$ steps to the right (and also, of course, $p$ steps to the left) is

$$
\left(\begin{array}{c}
n \\
2 p
\end{array}\right)\left[\left(\begin{array}{c}
2 p \\
p
\end{array}\right)-\left(\begin{array}{c}
2 p \\
p-1
\end{array}\right)\right]
$$

where the first factor is the number of ways to choose $2 p$ nonzero steps among the $n$ steps and the second factor is the number of walks of $2 p$ steps, with $p$ steps each to the right and left, which go from 1 to 1 without visiting 0 ; this number can be computed by the method of images. Thus

$$
\left\langle 1\left|C^{n}\right| 1\right\rangle=\sum_{p=0}^{[n / 2]}\left(\begin{array}{c}
n \\
2 p
\end{array}\right)(x+z)^{n-2 p}(x z)^{p}\left[\left(\begin{array}{c}
2 p \\
p
\end{array}\right)-\left(\begin{array}{c}
2 p \\
p-1
\end{array}\right)\right]
$$


Equation (A.3) may be rewritten in a convenient alternate form using the identity

$$
\sum_{j}\left(\begin{array}{c}
a \\
j
\end{array}\right)\left(\begin{array}{c}
b \\
j+c
\end{array}\right)=\left(\begin{array}{c}
a+b \\
a+c
\end{array}\right)
$$

which is easily verified by induction on $a$ from the fundamental identity

$$
\left(\begin{array}{c}
n+1 \\
m
\end{array}\right)=\left(\begin{array}{c}
n \\
m
\end{array}\right)+\left(\begin{array}{c}
n \\
m-1
\end{array}\right)
$$

as follows:

$$
\begin{aligned}
\left\langle 1\left|C^{n}\right| 1\right\rangle & =\sum_{p=0}^{[n / 2]}\left(\begin{array}{c}
n \\
2 p
\end{array}\right)(x+z)^{n-2 p}(x z)^{p} \frac{1}{p+1}\left(\begin{array}{c}
2 p \\
p
\end{array}\right) \\
& =\sum_{p=0}^{[n / 2]} \sum_{k=0}^{n-2 p}\left(\begin{array}{c}
n \\
2 p
\end{array}\right)\left(\begin{array}{c}
n-2 p \\
k
\end{array}\right) x^{k+p} z^{n-(k+p)} \frac{1}{p+1}\left(\begin{array}{c}
2 p \\
p
\end{array}\right) \\
& =\sum_{q=0}^{n} \frac{n !}{q !(n+1-q) !} x^{q} z^{n-q} \sum_{p}\left(\begin{array}{l}
q \\
p
\end{array}\right)\left(\begin{array}{c}
n+1-q \\
p+1
\end{array}\right) \\
& =\sum_{q=0}^{n} \frac{1}{q+1}\left(\begin{array}{c}
n+1 \\
q
\end{array}\right)\left(\begin{array}{l}
n \\
q
\end{array}\right) x^{q} z^{n-q}
\end{aligned}
$$

From this we may obtain the large- $n$ behavior of $\left\langle 1\left|C^{n}\right| 1\right\rangle$, for example, by calculating the contribution of the values of $q$ which dominate the sums:

$$
\left\langle 1\left|C^{n}\right| 1\right\rangle \simeq \frac{(\sqrt{x}+\sqrt{z})^{2 n+3}}{2 \sqrt{\pi} n^{3 / 2}(x z)^{3 / 4}}
$$

It will also be useful to have an expression for the generating function of the $\left\langle 1\left|C^{n}\right| 1\right\rangle$. From (A.3), for $|\lambda| \leqslant(\sqrt{x}+\sqrt{z})^{-2}$,

$$
\begin{aligned}
\sum_{n=0}^{\infty} \lambda^{n}\left\langle 1\left|C^{n}\right| 1\right\rangle & =\sum_{n=0}^{\infty} \sum_{p=0}^{[n / 2]}\left(\begin{array}{c}
n \\
n-2 p
\end{array}\right) \lambda^{n}(x+z)^{n-2 p}(x z)^{p} \frac{(2 p) !}{p !(p+1) !} \\
& =\sum_{p=0}^{\infty} \sum_{q=0}^{\infty}\left(\begin{array}{c}
2 p+q \\
q
\end{array}\right)(x+z)^{q} \lambda^{2 p+q}(x z)^{p} \frac{(2 p) !}{p !(p+1) !} \\
& =\sum_{p=0}^{\infty}[1-\lambda(x+z)]^{-2 p-1}\left(\lambda^{2} x z\right)^{p} \frac{(2 p) !}{p !(p+1) !} \\
& =\frac{1-\lambda(x+z)-\left\{[1-\lambda(x+z)]^{2}-4 \lambda^{2} x z\right\}^{1 / 2}}{2 \lambda^{2} x z}
\end{aligned}
$$

where we have used the standard Taylor series

$$
\frac{1-(1-4 u)^{1 / 2}}{2 u}=\sum_{p=0}^{\infty} \frac{(2 p) !}{p !(p+1) !} u^{p}
$$


The formula (A.7) simplifies at the radius of convergence: when $\lambda=(\sqrt{x}+\sqrt{z})^{-2}$,

$$
\sum_{n=0}^{\infty}\left\langle 1\left|C^{n}\right| 1\right\rangle(\sqrt{x}+\sqrt{z})^{-2 n}=\frac{(\sqrt{x}+\sqrt{z})^{2}}{\sqrt{x} \sqrt{z}}=\frac{1}{\rho(1-\rho)}
$$

where in the last step we have taken $\rho=\sqrt{x} /(\sqrt{x}+\sqrt{z})$, as in Sections 3 and 4.

We finally turn to the discussion of the matrix elements of $G=x D+$ $y A+z E$ defined in (6.1). It is convenient to begin with the calculation of the generating function of the $\left\langle 1\left|G^{n}\right| 1\right\rangle$. By writing $G=y A+C=$ $y|1\rangle\langle 1|+C$, we see that for $\lambda$ sufficiently small,

$$
\begin{aligned}
\sum_{n=0}^{\infty} \lambda^{n}\left\langle 1\left|G^{n}\right| 1\right\rangle & =\sum_{k=0}^{\infty}(\lambda y)^{k}\left[\sum_{n=0}^{\infty} \lambda^{n}\left\langle 1\left|C^{n}\right| 1\right\rangle\right]^{k+1} \\
& =\sum_{n=0}^{\infty} \lambda^{n}\left\langle 1\left|C^{n}\right| 1\right\rangle\left[1-\lambda y \sum_{n=0}^{\infty} \lambda^{n}\left\langle 1\left|C^{n}\right| 1\right\rangle\right]^{-1}
\end{aligned}
$$

We now claim that the $\left\langle 1\left|G^{n}\right| 1\right\rangle$ satisfy the recursion

$$
\left\langle 1\left|G^{n}\right| 1\right\rangle=\frac{(y+x)(y+z)}{y}\left\langle 1\left|G^{n-1}\right| 1\right\rangle-\frac{x z}{y}\left\langle 1\left|C^{n-1}\right| 1\right\rangle
$$

To verify this claim, we note that if (A.12) is satisfied, then

$$
\begin{aligned}
\sum_{n=0}^{\infty} \lambda^{n}\left\langle 1\left|G^{n}\right| 1\right\rangle= & {\left[1-\frac{(y+x)(y+z)}{y} \lambda\right]^{-1} } \\
& \times\left[1-\frac{x z}{y} \lambda \sum_{n=0}^{\infty} \lambda^{n}\left\langle 1\left|C^{n}\right| 1\right\rangle\right]
\end{aligned}
$$

and it is easy to see from the explicit formula (A.7) for the generating function of the $\left\langle 1\left|C^{n}\right| 1\right\rangle$ that (A.11) and (A.13) are identical. The recursion (A.12) together with the initial case $\left\langle 1\left|G^{0}\right| 1\right\rangle=1$ leads to

$$
\begin{aligned}
\left\langle 1\left|G^{n}\right| 1\right\rangle= & {\left[\frac{(y+x)(y+z)}{y}\right]^{n}\left[1-\frac{x z}{(y+x)(y+z)}\right.} \\
& \left.\times \sum_{q=0}^{n-1}\left(\frac{y}{(y+x)(y+z)}\right)^{q}\left\langle 1\left|C^{q}\right| 1\right\rangle\right]
\end{aligned}
$$

so that the explicit expression for the $\left\langle 1\left|G^{n}\right| 1\right\rangle$ may be obtained with the aid of either of the expressions (A.3), (A.5) for $\left\langle 1\left|C^{q}\right| 1\right\rangle$. Finally, we 
may find the asymptotic form of $\left\langle 1\left|G^{n}\right| 1\right\rangle$ directly from (A.14). We consider only the case $y^{2}>x z$ needed in Section 6; then taking $\lambda=y /[(y+x)(y+z)]$ in the generating function (A.7), we have

$$
\sum_{q=0}^{\infty}\left(\frac{y}{(y+x)(y+z)}\right)^{q}\left\langle 1\left|C^{q}\right| 1\right\rangle=\frac{(y+x)(y+z)}{y^{2}}
$$

so that (A.14) yields

$$
\left\langle 1\left|G^{n}\right| 1\right\rangle \simeq\left(1-\frac{x z}{y^{2}}\right)\left[\frac{(y+x)(y+z)}{y}\right]^{n}
$$

\section{ACKNOWLEDGMENTS}

This work was supported in part by NSF Grant DMR89-18903; S.A.J. was supported in part by NSF Mathematical Sciences Postdoctoral Research Fellowship DMS 90-07206. B.D. thanks the Mathematics Department of Rutgers University, where this work was initiated, for its hospitality, and S.A.J., J.L.L., and E.R.S. thank the IHES.

\section{NOTE ADDED IN PROOF}

It can be shown (see E. Speer, The two species totally asymmetric simple exclusion process, to appear in Micro, Meso, and Macroscopic Approaches in Physics, ed. M. Fannes, C. Maes, and A. Verbeure, Plenum) that the translation invariant steady state of Section 6 has exponential decay of correlations, is non-Gibbsian, and is the unique extremal state with densities $\rho_{1}, \rho_{2}$; the full proof of the Bernoulli property of first class particles to the right of a second class particle is also given in this article. We thank E. Andjel for pointing out the importance of some of these questions, and P. Ferrari for helpful comments on the manuscript.

\section{REFERENCES}

1. H. Spohn, Large-Scale Dynamics of Interacting Particles (Springer-Verlag, New York, 1991 ); A. De Masi and E. Presutti, Mathematical Methods for Hydrodynamic Limits (Springer-Verlag, New York, 1991); and references therein.

2. J. Lebowitz, E. Presutti, and H. Spohn, Microscopic models of hydrodynamic behavior, J. Stat. Phys. 51:841-862 (1988).

3. B. Schmittman, Critical behavior of the driven diffusive lattice gas, Int. J. Mod. Phys. B 4:2269-2306 (1990).

4. P. Garrido, J. Lebowitz, C. Maes, and H. Spohn, Long-range correlations for conservative dynamics, Phys. Rev. A 42:1954-1968 (1990).

5. R. Bhagavatula, G. Grinstein, Y. He, and C. Jayaprakash, Algebraic correlations in conserving chaotic systems, Phys. Rev. Lett. 69:3483-3486 (1992). 
6. T. M. Liggett, Interacting Particle Systems (Springer-Verlag, New York, 1985). See also T. M. Liggett, Ergodic theorems for the asymmetric simple exclusion process, Trans. Amer. Math. Soc. 213, 237-261 (1976), and T. M. Liggett, Ergodic theorems for the asymmetric simple exclusion process II, Ann. Prob. 5, 795-801 (1977).

7. H. Rost, Nonequilibrium behavior of many particle process: Density profiles and local equilibria, Z. Wahrsch. Verw. Gebiete 58:41-53 (1981).

8. A. Benassi and J. P. Fouque, Hydrodynamic limit for the asymmetric simple exclusion process, Ann. Prob. 15:546-560, and erratum. (1987).

9. E. D. Andjel and M. E. Vares, Hydrodynamical equations for attractive particle systems on $\mathbb{Z}$, J. Stat. Phys. 47:265-288 (1987).

10. D. Wick, A dynamical phase transition in an infinite particle system, J. Stat. Phys. 38:1015-1025 (1985).

11. P. Ferrari, The simple exclusion process as seen from a tagged particle, Ann. Prob. 14:1277-1290 (1986).

12. E. D. Andjel, M. Bramson, and T. M. Liggett, Shocks in the asymmetric exclusion process, Prob. Theory Related Fields 78:231-247 (1988).

13. A. De Masi, C. Kipnis, E. Presutti, and E. Saada, Microscopic structure at the shock in the asymmetric simple exclusion, Stoch. Stoch. Rep. 27:151-165 (1989).

14. P. Ferrari, C. Kipnis, and E. Saada, Microscopic structure of traveling waves in the asymmetric simple exclusion, Ann. Prob. 19:226-244 (1991).

15. P. Ferrari, Shock fluctuations in asymmetric simple exclusion, Prob. Theory Related Fields 91:81-101 (1992). See also P. A. Ferrari and L. R. G. Fontes, Shock fluctuations in the asymmetric simple exclusion process (1993), to appear in Prob. Theory Related Fields.

16. C. Boldrighini, G. Cosimi, S. Frigio, and M. G. Nuñes, Computer simulation of shock waves in the completely asymmetric simple exclusion process, J. Stat. Phys. 55:611-623 (1989).

17. S. A. Janowsky and J. L. Lebowitz, Finite size effects and shock fluctuations in the asymmetric simple exclusion process, Phys. Rev. A 45:618-625 (1992).

18. B. Derrida, S. A. Janowsky, J. L. Lebowitz, and E. R. Speer, Microscopic shock profiles: Exact solution of a nonequilibrium system, Europhys. Lett. 22 (1993).

19. B. Derrida, M. R. Evans, V. Hakim, and V. Pasquier, An exact solution of a 1D asymmetric exclusion model using a matrix formulation, J. Phys. A 26:1493-1517 (1993).

20. B. Derrida, E. Domany, and D. Mukamel, An exact solution of a one dimensional asymmetric exclusion model with open boundaries, J. Stat. Phys. 69:667-687 (1992). 